\title{
Regulatory effects of HIF-1alpha on eNOS expressions in the vascular endothelium of pre-diabetic rats with aerobic exercise.
}

\author{
Shaobing Wang ${ }^{1,2}$, Yedong Tang ${ }^{3}$, Zhenghong Zhang ${ }^{1,2,3}$, Zonghao Tang ${ }^{3}$, Yiping Liu ${ }^{1,2^{*}}$, Zhengchao \\ Wang ${ }^{1,2,3^{*}}$ \\ ${ }^{1}$ Laboratory of Sport Physiology and Biomedicine, School of Physical Education and Sport Sciences, Fujian Normal \\ University, Fuzhou, PR China \\ ${ }^{2}$ State Key Laboratory for Evaluation of Exercise Physiological Functions from General Administration of Sport of \\ China, Fujian Normal University, Fuzhou, PR China \\ ${ }^{3}$ Provincial Key Laboratory for Developmental Biology and Neurosciences, College of Life Sciences, Fujian Normal \\ University, Fuzhou, PR China
}

\begin{abstract}
Our previous study has demonstrated aerobic exercise contributed to the improvement of endothelial vasodilation functions by nitric oxide (NO) signaling activation during pre-diabetes mellitus. The present study was designed to examine the contribution of hypoxia inducible factor (HIF)-1alpha to the regulation of endothelial nitric oxide synthase (eNOS) expressions in the vascular endothelium of prediabetic rats with HIF-1alpha specific inhibitor echinomycin (Ech) and/or aerobic exercise. The results showed the expressions of eNOS mRNA and protein dramatically decreased after Ech treatment $(p<0.05)$, while significantly increased exercise $(\mathbf{p}<0.05)$. Interestingly, no obvious changes of HIF-1alpha mRNA and protein expressions were found after Ech treatment. Further investigation found HIF-1 binding activity was inhibited after Ech treatment, which may contribute to the decrease of eNOS expressions. Together, the present study clearly indicated aerobic exercise induced eNOS expressions through the activation of HIF-1alpha signaling in the vascular endothelium of rat aorta during prediabetes mellitus, which shed a light on further understanding the protective effects of long-term habitual physical activity against vasodilation dysfunctions during pre-diabetes mellitus.
\end{abstract}

Keywords: Aerobic exercise, Hypoxia inducible factor-1alpha, Endothelial nitric oxide synthase, Vascular endothelium, Pre-diabetes mellitus.

Accepted on August 07, 2018

\section{Introduction}

Pre-diabetes mellitus is a strong risk factor for the development of type 2 diabetes mellitus. Incidence of pre-diabetes is increasing in young people and the annual probability of prediabetes converted to diabetes is about $5 \% \sim 10 \%[1,2]$. The intact vascular endothelium prevented the injury on blood vessel under physiological conditions, while hyperglycemia was connected with the development of the endothelial dysfunction in diabetes mellitus [3-6]. Our previous reports have demonstrated aerobic exercise may improve the impaired endothelium-dependent vasodilatation in middle-aged patients with impaired glucose tolerance [3-5]. Our recent investigation showed that effects of aerobic exercise on expressions and activities of NOS in the blood vessel endothelium during prediabetes mellitus, but the regulatory mechanism of eNOS/NO signaling activation in the vascular endothelium of prediabetic rats with aerobic exercise still remains unclearly.
It is particularly noteworthy that HIF-1 as an important transcriptional factor can activate the transcription of many genes and then participate in various pathophysiological processes, including angiogenesis, glucose metabolism, inflammation, cell survival, and proliferation [7-14]. HIF-1 consists of HIF-1alpha and HIF-1beta, HIF-1alpha is an inducible protein while HIF-1beta is not inducible, but it can be bound to HIF-1alpha to form heterologous dimer to activate the transcription of target genes [7-12]. Paula et al. indicate that eccentric exercise promotes a significant activation of HIF-1alpha, which in turn contributes to the upregulation of eNOS expression in rat skeletal muscle [15]. Liang et al. demonstrated that HIF-1 is required in Tongxinluo-induced activation of eNOS/NO system, thus improving the vascular endothelial function [16]. These findings indicating HIF-1alpha may play an important role in the regulation of eNOS expression in the vascular endothelium of prediabetic rats during aerobic exercise. 
Therefore, the present study used a small molecule inhibitor of HIF-1alpha echinomycin (Ech) to further investigate the contribution of hypoxia inducible factor (HIF)-1alpha to the regulation of endothelial nitric oxide synthase (eNOS) expressions in the vascular endothelium of prediabetic rats with aerobic exercise, which will help us to further understand the protective effects and molecular mechanism of long-term habitual physical activity against vasodilation dysfunctions during pre-diabetes mellitus.

\section{Materials and Methods}

\section{Animals}

Sprague-Dawley rats (2 $\mathrm{m}$ old) are purchased from Wushi Experimental Animal Supply Co. Ltd. (Fuzhou, China). The animals are maintained under a $12 \mathrm{~h} \mathrm{light} / 12 \mathrm{~h}$ dark schedule at $20-25^{\circ} \mathrm{C}$, with a continuous supply of chow and water. The experimental protocol (No. IACUC-20160019) is approved in accordance with the Guide for the Care and Use of Laboratory Animals prepared by the Institutional Animal Care and Use Committee, Fujian Normal University.

\section{Experiment design}

To examine the effect of Ech on eNOS expressions in the vascular endothelium of prediabetic rats, the animals were are administered by a fixed dose echinomycin (i.p., $0.1 \mathrm{mg} / \mathrm{kg}$ body weight, Tocris Bioscience, Bristol, United Kingdom). Firstly, 24 prediabetic rats were developed by an additional high-energy diet, as previously described [17]. Briefly, rats were daily given a high-energy emulsion for one month. Animals were progressively administered 1-5 $\mathrm{ml}$ of emulsion in the first $5 \mathrm{~d}$ and then were administered $5 \mathrm{ml}$ of emulsion. After comprehensive analysis of glucose and AUCg, these prediabetic rats were ready for the following experiments. Secondly, these experimental rats are randomly divided into 4 groups, including the sedentary control group, aerobic exercise group, Ech treatment group and aerobic exercise plus Ech treatment group. Finally, $24 \mathrm{~h}$ after the last bout of aerobic exercise training, the animals are anesthetized with an i.p. injection of pentobarbital solution (Sigma-Aldrich, Shanghai, China) and sacrifice by cervical dislocation. The thoracic aorta is rapidly removed and cleaned from all loosely adherent tissues. The partial aorta is preserved in 4\% paraformldehyde for histological sections. The remaining aorta is snap frozen in liquid nitrogen and store in $\mathrm{a}-80^{\circ} \mathrm{C}$ for additional analyses.

\section{Exercise training}

Aerobic exercise training is carried out according to our previous research. Briefly, all rats are acclimated to treadmill running (10 min, using different speeds) for 1 week. On the first day, an electric shock is applied to make the rats start running, after which they can run spontaneously. The maximal aerobic velocity (MAV) is evaluated with an incremental test to exhaustion using a protocol with an initial velocity of $5 \mathrm{~m} / \mathrm{min}$ being intensified every $5 \mathrm{~min}$ with a speed of $5 \mathrm{~m} / \mathrm{min}$, until the moment when the animal is unable or unwilling to continue. Aerobic exercise training is performed on a treadmill at low-moderate intensity (50\%-60\% MAV), $1 \mathrm{~h}$ per day, and 5 $\mathrm{d}$ per week for 8 weeks. The experiment is carried out in a quiet, well-ventilated room with low humidity, at room temperature around $18^{\circ} \mathrm{C} \pm 2^{\circ} \mathrm{C}$. Sedentary rats in the control group are handled the same as the exercised rats except no engaging in the regular running.

\section{RNA extraction and real-time PCR analysis of eNOS and HIF-1alpha mRNA}

Total RNA is extracted from the aortas using TRIzol solution (Life Technologies, Carlsbad, CA, USA), quantified by Nanodrop 2000 (Thermo, Wilmington, USA) and reverse transcribed into cDNA using a FastKing One Step RT-PCR Kit (Tiangen, Beijing, China), according to the manufacturer's instructions. Real-time PCR is performed to analyze eNOS and HIF-1alpha mRNA levels, according to the standard protocol of the GoTaq ${ }^{\circledR}$ qPCR Master Mix for Dye-Based Detection kit (Promega, Beijing, China). The primers are synthesized by Takara Biotech Co. (Takara, Dalian, China), including eNOS primer (F: 5'-GGC AGA GGA GTC CAG CGA AC-3', R: 5'TGT GGA ACA GAC CCC ATA GTG C-3'), HIF-1alpha primer (F: 5'-GCG AGA ACG AGA AGA AAA ATA GG-3', R: 5'-AGT TCA TCT GTG CTT TCA TTT CAT C-3') and GADPH primer (5'-CGA CCC CTT CAT TGA CCT CAA C-3', R: 5'-AAG ACG CCA GTA GAC TCC ACG AC-3'). The amount of target gene mRNA relative to the internal control gene, GADPH, is calculated in accordance with the $\Delta \Delta \mathrm{Ct}$ method. Relative mRNA levels are reported as $2^{-\Delta \Delta \mathrm{Ct}}$ values. The results of three independent experiments are used for statistical analysis.

\section{Immunohistochemical analysis of eNOS and HIF-1alpha}

After fixation by the conventional procedures, aortic segments were embedded in paraffin and then section into $5 \mu \mathrm{m}$ slides. Immunohistochemical analysis of eNOS and HIF-1alpha was performed by anti-eNOS antibody (1:1000, Abcam, Cambridge, MA, USA; Cat No: ab195944) and antiHIF-1alpha antibody (1:500, Santa Cruz, CA, USA; Cat No: sc-53546); the sections are incubated at room temperature overnight with primary antibody. The immunoreactivity of the specific protein was visualized by Elite ABC kit (BioGenex, San Ramon, CA, USA). Then the sections were counterstained with hematoxylin and mounted with cover slips to identify the structure and type of cells in the rat aorta. The negative control used serum (Boster Biological Technology, WuhanChina) instead of primary antibody and then these slides were examined under microscope. To assign the intensities of staining for eNOS and HIF-1alpha, three independent observers blinded to the experimental design were asked to examine the pictures, using a method previously described [18]: -, no staining detected; +, weak; ++, moderate; +++ , strong staining. All observers evaluated all slides, and observations outside the $5 \%-95 \%$ of the remaining observations for the treatment group were considered outlying 
data and excluded from analysis. Relative levels of immunostaining were evaluated and repeated at least four times. The results represent consistently observed patterns of immunohistochemistry.

\section{Western blot analysis of eNOS and HIF-1alpha expressions}

The Western blot procedure is performed as previously described [7-12,19]. Following transfer, the membranes are incubated with anti-eNOS antibodies (1:1000 dilution; Abcam, Cambridge, MA, USA; Cat No: ab195944) or anti-HIF-1alpha antibody (1:500; Santa Cruz, CA, USA; Cat No: sc-53546). Anti-.-actin antibody (1:1000 dilution; Beyotime Inc., Jiangsu, China; Cat No: AF0003) was used as the internal loading control. Membranes are detected with BeyoECL Star Western blot detection reagent (Beyotime Inc., Jiangsu, and China). The relative intensity of eNOS or HIF-1alpha compared to .-actin was quantified using the AlphaView ${ }^{\circledR}$ Q software (Proteinsimple, San Jose, California, USA).

\section{Extraction of nuclear proteins and analysis of HIF banding activity}

Nuclear protein is prepared by a nuclear extract kit (Panomics, Fremont, CA), according to the manufacturer's instructions. Briefly, the tissue was diced and homogenized on ice, $1.5 \mathrm{~mL}$ Buffer A was then added and homogenized again for centrifuging at $850 \times \mathrm{g}$ for $10 \mathrm{~min}$ at $4^{\circ} \mathrm{C}$ after incubating on ice for $15 \mathrm{~min}$. Discarded the supernatant and repeated above steps for lysing most of cells. $150 \mathrm{uL}$ of Buffer B was added into the tube of the pellet after centrifuging at $14,000 \times \mathrm{g}$ for 3 $\min$ at $4{ }^{\circ} \mathrm{C}$, vortexed, incubated on ice for $60 \mathrm{~min}$, and gently agitated the tubes by hand every $20 \mathrm{~min}$. The supernatant (nuclear protein) was acquired by centrifugation at $14,000 \times \mathrm{g}$ for $5 \mathrm{~min}$ at $4^{\circ} \mathrm{C}$. The protein concentration is detected by the BCA protein assay kit (Beyotime Inc., Jiangsu, and China) and nuclear protein is stored at $-80^{\circ} \mathrm{C}$. HIF-1 binding activities in the nuclear extracts were detected by an ELISA-based HIF binding kit (Panomics, Fremont, CA). The ELISA-based HIF binding assay kit provides a fast, sensitive, and specific measurement for the HIF-1 binding activities.

\section{Statistical analysis}

Data are presented as the mean \pm SEM. The significance of differences in mean values between multiple groups was evaluated by one-way ANOVA, followed by a Tukey's multiple range test (SPSS version 19.0, SPSS Inc., Chicago, IL, USA). $\mathrm{P}<0.05$ is considered statistical significance. Different superscript letters denoted significant values $(\mathrm{p}<0.05)$.

\section{Results}

\section{Effect of Ech on eNOS mRNA expression in the vascular endothelium of prediabetic rats with aerobic exercise}

Given the role of eNOS in the vasodilation dysfunctions during pre-diabetes mellitus, the present study firstly examined eNOS mRNA expression in the vascular endothelium of each group by real time PCR (Figure 1). The results showed an obvious increase of eNOS mRNA expression after exercise intervention $(p<0.05$, Figure 1) and a significant decrease of eNOS mRNA expression after Ech treatment compared with each control $(\mathrm{p}<0.05$, Figure 1), indicating exercise intervention induced increase of eNOS mRNA expression was inhibited by HIF-1alpha specific inhibitor Ech.

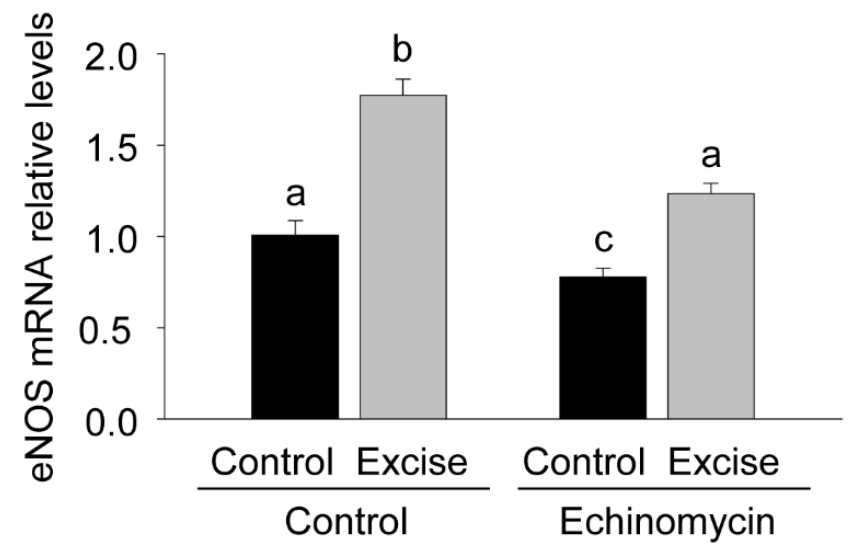

Figure 1. eNOS mRNA expression levels in in the vascular endothelium of prediabetic rats with aerobic exercise. The expression of eNOS mRNA was examined by real-time PCR. $n=6$. One-way analysis of variance (ANOVA) was used to analyze the data. Different superscripts denote significant values between the other groups $(p<0.05)$ by Tukey's multiple range test.

\section{Immunohistochemical localization and expression of eNOS protein in the vascular endothelium of prediabetic rats with aerobic exercise}

For further confirming the pattern of eNOS expression, the present study secondly examined the expression of eNOS protein in the vascular endothelium of each group by immunostaining (Figure 2). The results showed the positive immunostaining for eNOS decreased in the endothelial and smooth muscle layers of the aorta in the Ech treatment group compared with the sedentary control group (Figures 2A and $2 \mathrm{C}$, Table 1) and the positive signal was stronger than that after exercise intervention before in these two groups (Figures 2B and 2D, Table 1), implying HIF-1alpha may pay an important role in the regulation of eNOS expression in the vascular endothelium of prediabetic rats with exercise intervention. 


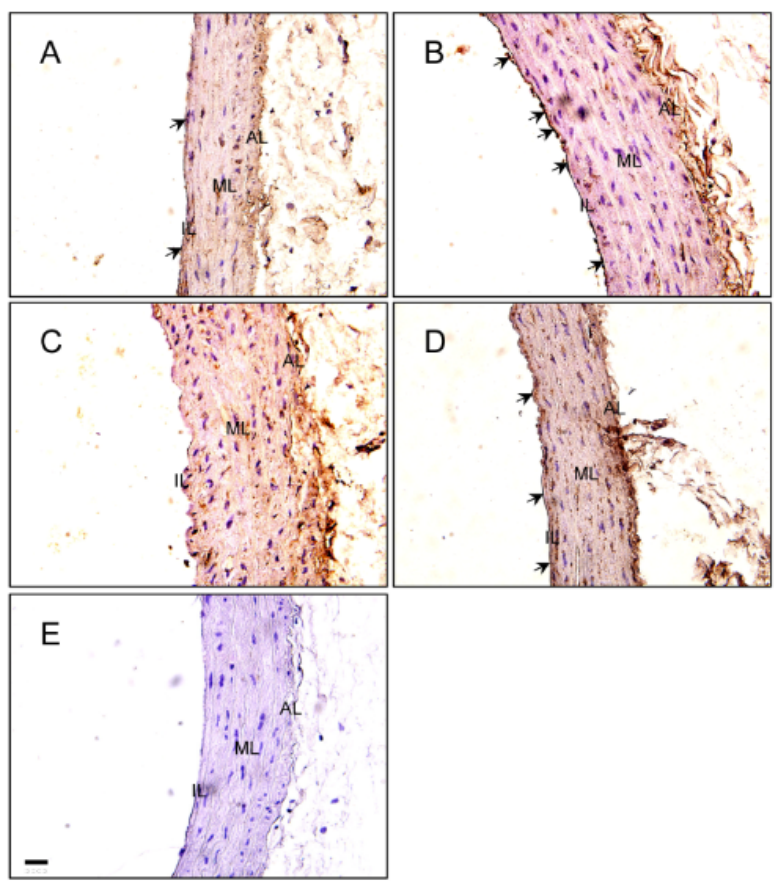

Figure 2. Expression and localization of eNOS protein in the vascular endothelium of prediabetic rats with aerobic exercise. eNOS immunohistochemical signals appear brown and the counterstaining background appears blue in color $(A, B, C$ and $D)$. Negative controls remained unstained lacking primary antibody instead of serum (E). A: Sedentary control group; B: aerobic exercise group; $C$ : Ech treatment group; D: Aerobic exercise plus Ech treatment group; E: Negative control group; AL: Adventitia Layer; ML: Medial Layer; IL: Intimal Layer; Bar $=100$ um.

\section{Expression changes of eNOS protein in the vascular endothelium of prediabetic rats with aerobic exercise}

For quantitative analysis of eNOS expression, the present study thirdly examined the expression level of eNOS protein by Western blotting (Figure 3). The results showed eNOS expression significantly increased after exercise intervention $(\mathrm{p}<0.05$, Figures $3 \mathrm{~A}$ and $3 \mathrm{~B})$, which was inhibited by HIF-1alpha specific inhibitor Ech $(\mathrm{p}<0.05$, Figures $3 \mathrm{~A}$ and 3B), further suggesting HIF-1alpha was involved in the regulation of eNOS transcription.

\section{Effect of Ech on HIF-1alpha mRNA expression in the vascular endothelium of prediabetic rats with aerobic exercise}

For clarifying the regulatory effect of HIF-1alpha on eNOS transcription, the present study firstly examined HIF-1alpha mRNA expression in the vascular endothelium of each group by real time PCR (Figure 4).

The results showed a significant increase of HIF-1alpha mRNA expression after exercise intervention $(\mathrm{p}<0.05$, Figure 4) and no obvious changes after Ech treatment compared with each control ( $p>0.05$, Figure 4$)$, indicating there was no effect of HIF-1alpha specific inhibitor Ech on HIF-1alpha mRNA expressions.
A
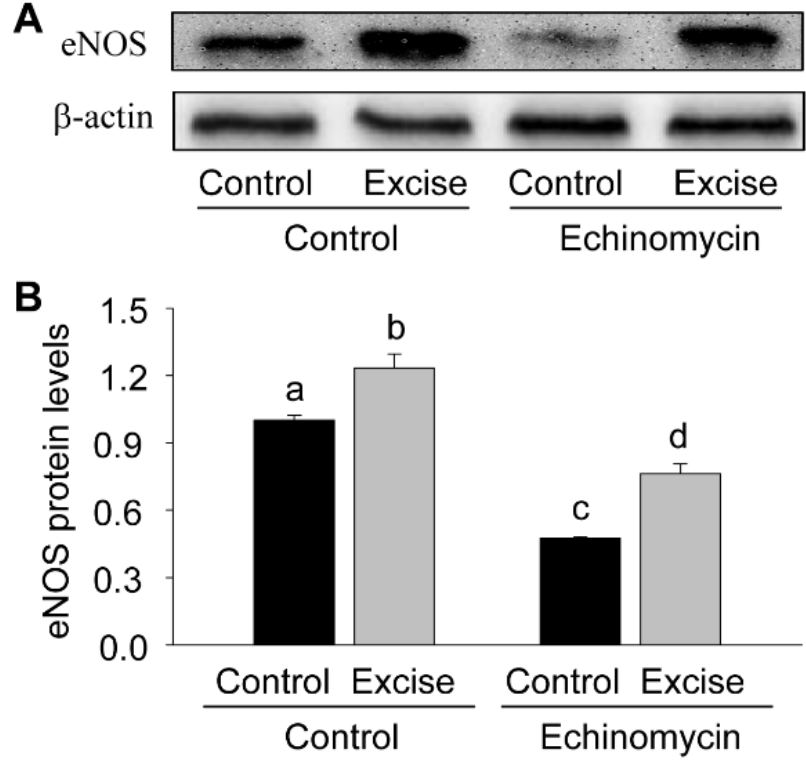

Figure 3. Blotting analysis of eNOS protein in the vascular endothelium of prediabetic rats with aerobic exercise. Panel A: Representative ECL gel documents of Western blot analyses depicting the protein levels of eNOS. Panel B: Summarized intensities of eNOS blots normalized to the control. Each value represents the mean $\pm S E$, $n=6$. One-way analysis of variance (ANOVA) was used to analyze the data. Different superscripts denote significant values between the other groups $(p<0.05)$ by Tukey's multiple range test.

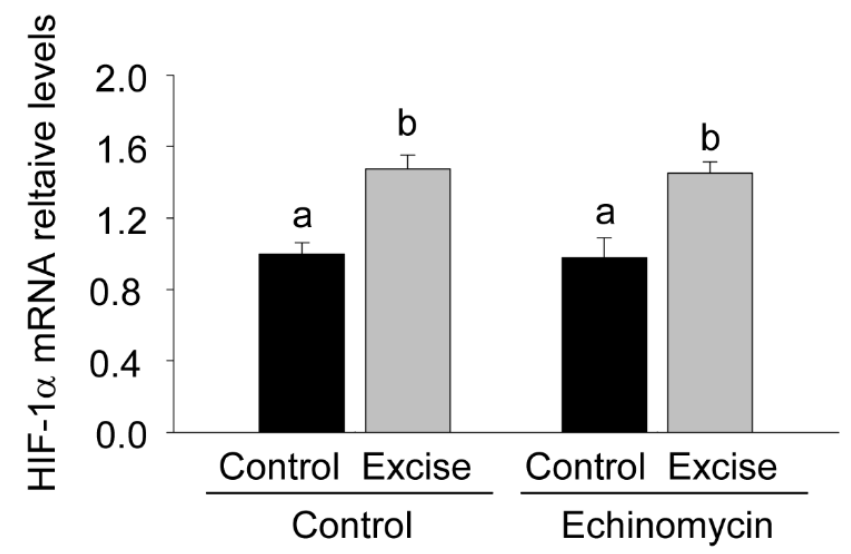

Figure 4. HIF-1alpha mRNA expression levels in in the vascular endothelium of prediabetic rats with aerobic exercise. The expression of HIF-1alpha mRNA was examined by real-time PCR. $n=6$. One-way analysis of variance (ANOVA) was used to analyze the data. Different superscripts denote significant values between the other groups $(p<0.05)$ by Tukey's multiple range test.

\section{Immunohistochemical localization and expression of HIF-1alpha protein in the vascular endothelium of prediabetic rats with aerobic exercise}

The present study also detected HIF-1alpha expression by immunohistochemistry (Figure 5), the results showed a significant increase of HIF-1alpha protein expression after exercise intervention (Figures 5A and 5C and Table 1) and no obvious changes after Ech treatment compared with each control (Figure 6 and Table 1), indicating there was no effect of 
Regulatory effects of HIF-1alpha on eNOS expressions in the vascular endothelium of pre-diabetic rats with aerobic exercise

HIF-1alpha specific inhibitor Ech on HIF-1alpha protein expressions.

Table 1. Relative levels of eNOS and HIF-1alpha immunostaining in the vascular endothelium of pre-diabetic rats with aerobic exercise.

\begin{tabular}{lll}
\hline Experiment groups & eNOS IHC & HIF-1alpha IHC \\
\hline Group A & + & + \\
\hline Group B & +++ & ++ \\
\hline Group C & - & + \\
\hline Group D & ++ & ++ \\
\hline Group E & - & -
\end{tabular}

Note: A: Sedentary control group; B: Aerobic exercise group; C: Ech treatment group; D: Aerobic exercise plus Ech treatment group; E: Negative control group.

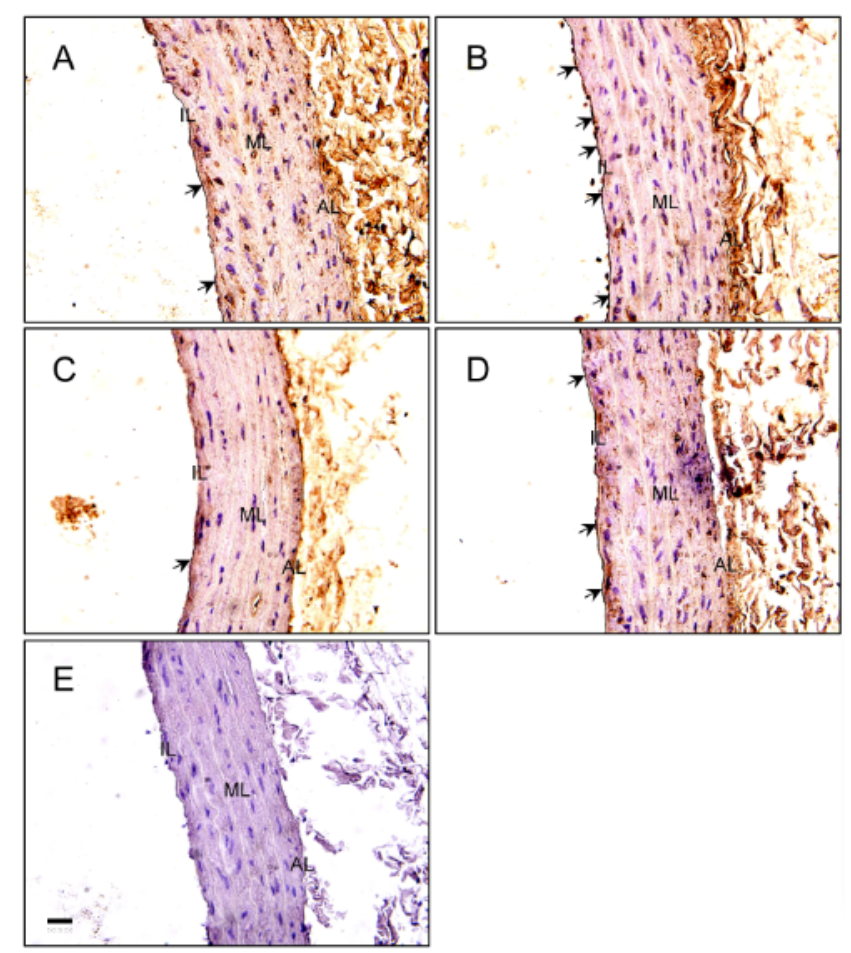

Figure 5. Expression and localization of HIF-1alpha protein in the vascular endothelium of prediabetic rats with aerobic exercise. HIF-1alpha immunohistochemical signals appear brown and the counterstaining background appears blue in color (A, B, C and D). Negative controls remained unstained lacking primary antibody instead of serum (E). A: Sedentary control group; B: Aerobic exercise group; C: Ech treatment group; D: Aerobic exercise plus Ech treatment group; E: Negative control group; AL: Adventitia Layer; ML: Medial Layer; IL: Intimal Layer. Bar=100 um.

\section{Expression changes of HIF-1alpha protein in the vascular endothelium of prediabetic rats with aerobic exercise}

For quantitative analysis of HIF-1alpha expression, the present study thirdly examined the expression level of HIF-1alpha protein by Western blotting (Figure 6). The results showed HIF-1a expression significantly increased after exercise intervention $(\mathrm{p}<0.05$, Figures $6 \mathrm{~A}$ and $6 \mathrm{~B})$ and no obvious changes were found after Ech treatment compared with each control ( $p>0.05$, Figures $6 \mathrm{~A}$ and $6 \mathrm{~B})$, which was consist with the changes of HIF-1a mRNA expression, further indicating there was no effect of HIF-1alpha specific inhibitor Ech on HIF-1alpha expressions.

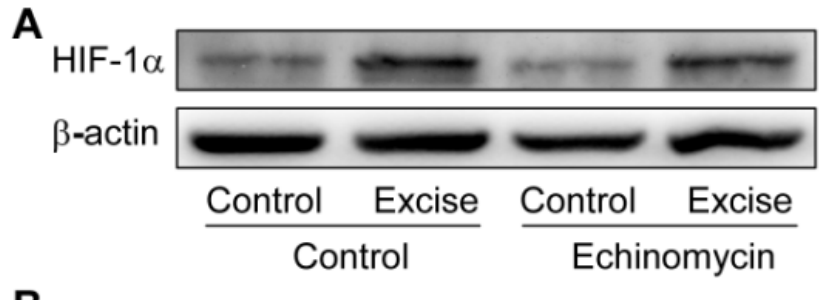

B

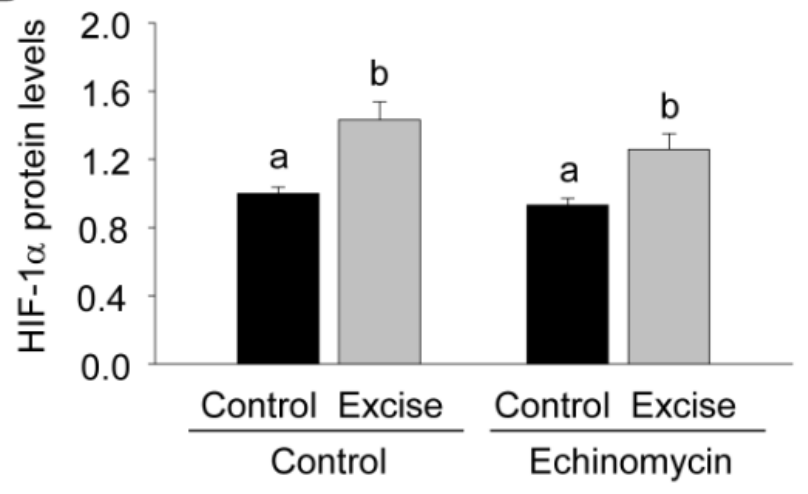

Figure 6. Blotting analysis of HIF-1alpha protein in the vascular endothelium of prediabetic rats with aerobic exercise. Panel A: Representative ECL gel documents of Western blot analyses depicting the protein levels of HIF-1alpha. Panel B: Summarized intensities of HIF-lalpha blots normalized to the control. Each value represents the mean $\pm S E, n=6$. One-way analysis of variance (ANOVA) was used to analyze the data. Different superscripts denote significant values between the other groups $(p<0.05)$ by Tukey's multiple range test.

\section{Effect of Ech on HIF-1 binding activities in the vascular endothelium of prediabetic rats with aerobic exercise}

Given Ech as an inhibitor of HIF-1 binding activity, the present study further examined the effect of Ech on HIF-1 binding activities in the vascular endothelium of prediabetic rats with aerobic exercise. The results showed HIF-1 binding activity significantly increased after exercise intervention $(p<0.05$, Figure 7), which was inhibited by HIF-1alpha specific inhibitor Ech ( $p<0.05$, Figure 7), which was consist with the changes of eNOS expression, demonstrating the regulatory role of HIF-1alpha in eNOS expression in the vascular endothelium of prediabetic rats with exercise intervention.

\section{Discussion}

The present study clearly demonstrated the expressions of eNOS and HIF-1alpha in the vascular endothelium and the regulation of HIF-1alpha on eNOS expression in prediabetic rats with aerobic exercise, which will help us to further 
understand the mechanism of exercise intervention improving the vascular functions in prediabetic rats.

Nitric oxide (NO) is the key transmitter for endotheliumdependent regulation of vascular tone in the arterial wall, and is produced in endothelial cells by the eNOS [20] after the activation of the hypoxia response element (HRE) in the eNOS promoter by the transcript factor HIF-1alpha/beta [21].

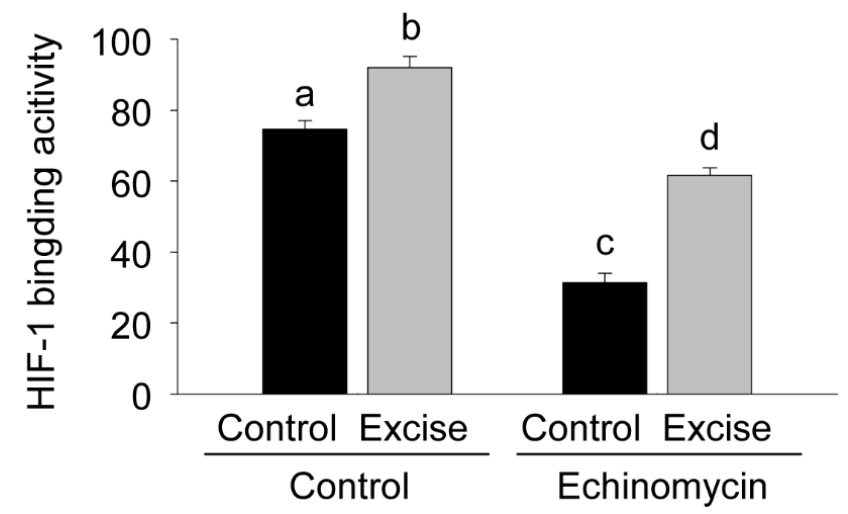

Figure 7. Analysis of HIF-1 binding activity in the vascular endothelium of prediabetic rats with aerobic exercise. Nuclear protein was extracted by a nuclear extract kit and HIF-1 binding activities in the nuclear extracts are detected by an ELISA-based HIF binding kit. The ELISA-based HIF binding assay kit provides a fast, sensitive, and specific measurement for the HIF-1 binding activities. $n=6$. One-way analysis of variance (ANOVA) was used to analyze the data. Different superscripts denote significant values between the other groups $(p<0.05)$ by Tukey's multiple range test.

Our previous study showed that aerobic exercise improved the impaired endothelium-dependent vasodilatation in middle-aged prediabetic patients [4-6], and animal studies of exercise training in prediabetic rats demonstrated aerobic exercise also improved the vascular endothelial dysfunctions by activating eNOS/NO system in the aorta, but the upstream components of eNOS/NO pathway need further investigation.

At present, HIF-1alpha is a transcript factor, which plays an important role in the regulation of many pathophysiological processes through activating its target gene expressions [7-16]. In the present study, HIF-1alpha inhibitor Ech was used to investigate the regulatory mechanism of eNOS/NO signaling in the vascular endothelium of prediabetic rats with exercise intervention. The present findings indicated the endotheliumdependent vasodilation was improved by aerobic exercise through the activation of eNOS signaling, which was also inhibited by Ech treatment. These findings were consistent with previous researches [15-17,19-25] and indicated the regulatory role of HIF-1alpha in eNOS expression in the vascular endothelium of prediabetic rats with exercise intervention. Previous study demonstrated the endotheliumdependent vasodilation was improved by Tongxinluo through HIF/eNOS pathway [16], the other study indicated HIF-1alpha binds to the HRE region of eNOS promoter after acute eccentric exercise in rat skeletal muscle [15]. Moreover, hyperglycaemia increased methylglyoxal-modification of HIF-1alpha, which interfered with the dimerization of
HIF-1alpha and HIF-1beta and with the binding of HIF-1 to the HRE-containing regions of HIF-1 target genes in diabetes [22]. Ech is a small molecule inhibitor, which specifically inhibited binding of HIF-1 to the HRE sequence contained in the promoter regions of target genes [23,24]. Together, HIF-1alpha may contribute to a larger release of NO by activating eNOS [25] and promote the changes in the vascular tone [20].

In summary, the present study clearly demonstrated that aerobic exercise promoted a significant activation of HIF-1alpha signaling in the vascular endothelium and resulted in the increased expression of eNOS. The experiment of Ech treatment further indicated the regulatory effect of HIF-1alpha on eNOS expression in the vascular endothelium of prediabetic rats with aerobic exercise, which shed a light on further understanding the protective effects of long-term habitual physical activity against vasodilation dysfunctions during prediabetes mellitus.

\section{Acknowledgement}

This study was supported by Fujian Provincial Natural Science Foundation (2016J01145, 2016J01150 and 2017J01627) and Fujian Province Science and Technology Project of The Education Department (JAT160118 and JZ160426).

\section{Disclosure of Conflict of Interest}

None.

\section{References}

1. American Diabetes Association. Diagnosis and classification of diabetes mellitus. Diabetes Care 2012; 35: 64-71.

2. Tabák AG, Herder C, Rathmann W, Brunner EJ, Kivimäki M. Pre-diabetes: a high-risk state for diabetes development. Lancet 2012; 379: 2279-2290.

3. Liu Y, Li J, Zhang Z, Tang Y, Chen Z, Wang Z. Effects of exercise intervention on vascular endothelium functions of impaired glucose tolerance patients during Pre-diabetes mellitus. Exp Ther Med 2013; 5: 1559-1565.

4. Liu Y, Li J, Zhang Z, Tang Y, Chen Z, Wang Z. Endocrinological analysis of endothelium-dependent vasodilation in middle-aged patients with impaired glucose tolerance during pre-diabetes mellitus. Exp Ther Med 2014; 7: 697-702.

5. Liu Y, Zhang Z, Li J, Wang S, Chen J, Wang Z. Changes of leptin, resistin, adiponectin and free fatty acid in the impaired glucose tolerance patients with exercise intervention. Biomed Res 2017; 28: 6241-6247.

6. Fiorentino TV, Prioletta A, Zuo P, Folli F. Hyperglycemiainduced oxidative stress and its role in diabetes mellitus related cardiovascular diseases. Curr Pharm Des 2013; 19: 5695-5703.

7. Zhang Z, Yin D, Wang Z. Contribution of hypoxiainducible factor- $1 \alpha$ to transcriptional regulation of 
vascular endothelial growth factor in bovine developing luteal cells. Anim Sci J 2011; 82: 244-250.

8. Zhang Z, Yu D, Yin D, Wang Z. Activation of PI3K/ mTOR signaling pathway contributes to induction of vascular endothelial growth factor by $\mathrm{hCG}$ in bovine developing luteal cells. Anim Reprod Sci 2011; 125: 42-48.

9. Wu L, Zhang Z, Pan X, Wang Z. Expression and contribution of the HIF-1a/VEGF signaling pathway to luteal development and function in pregnant rats. Mol Med Rep 2015; 12: 7153-7159.

10. Wang F, Zhang Z, Wang Z, Xiao K, Wang Q, Su J, Wang $Z$. Expression and clinical significance of the HIF-1a/ET-2 signaling pathway during the development and treatment of polycystic ovary syndrome. J Mol Histol 2015; 46: 173-181.

11. Wang Z, Zhu Q, Li PL, Dhaduk R, Zhang F, Gehr TW, Li $\mathrm{N}$. Silencing of hypoxia-inducible factor-1 $\alpha$ gene attenuates chronic ischemic renal injury in two-kidney, one-clip rats. Am J Physiol Renal Physiol 2014; 306: 1236-1242.

12. Zhang J, Zhang Z, Wu Y, Chen L, Luo Q, Chen J, Huang X, Cheng Y, Wang Z. Regulatory effect of hypoxiainducible factor- $1 \alpha$ on hCG-stimulated endothelin-2 expression in granulosa cells from the PMSG-treated rat ovary. J Reprod Dev 2012; 58: 678-684.

13. Albina JE, Mastrofrancesco B, Vessella JA, Louis CA, Henry WL Jr, Reichner JS. HIF-1 expression in healing wounds: HIF-1alpha induction in primary inflammatory cells by TNF-alpha. Am J Physiol Cell Physiol 2001; 281: 1971-1977.

14. Semenza GL. Expression of hypoxia-inducible factor 1: mechanisms and consequences. Biochem Pharmacol 2000; 59: 47-53.

15. Rodriguez-Miguelez P, Lima-Cabello E, Martínez-Flórez S, Almar M, Cuevas MJ, González-Gallego J. Hypoxiainducible factor-1 modulates the expression of vascular endothelial growth factor and endothelial nitric oxide synthase induced by eccentric exercise. J Appl Physiol 2015; 118: 1075-1083.

16. Liang J, Wu K, Jia Z, Liu C, Ding J, Huang S, Yin P, Wu $\mathrm{X}$, Wei $\mathrm{C}$, Wu Y, Wang $\mathrm{H}$. Chinese medicine Tongxinluo modulates vascular endothelial function by inducing eNOS expression via the PI-3K/Akt/HIF-dependent signaling pathway. J Ethnopharmacol 2011; 133: 517-523.

17. Rato L, Duarte AI, Tomás GD, Santos MS, Moreira PI, Socorro S, Cavaco JE, Alves MG and Oliveira PF: Prediabetes alters testicular PGC1-a/SIRT3 axis modulating mitochondrial bioenergetics and oxidative stress. Biochim Biophys Acta 2014; 1837: 335-344.

18. Wei Q, Shi F, He J, Xie C, Xu K, Zhang W, Sun S, Fedail J, Watanabe G, Taya K. Effects of exogenous $17 \beta$ - estradiol on follicular development in the neonatal and immature mouse in vivo. Reprod Med Biol 2012; 11: 135-141.

19. Wang S, Xing B, Yi L, Wang W, Xu Y. Expression of Frizzled 2 in the mouse ovary during oestrous cycle. J Anim Physiol Anim Nutr (Berl) 2010; 94: 437-445.

20. Gielen S, Sandri M, Erbs S, Adams V. Exercise-induced modulation of endothelial nitric oxide production. Curr Pharm Biotechnol 2011; 12: 1375-1384.

21. Coulet F, Nadaud S, Agrapart M, Soubrier F. Identification of hypoxia-response element in the human endothelial nitric-oxide synthase gene promoter. J Biol Chem 2003; 278: 46230-46240.

22. Ceradini DJ, Yao D, Grogan RH, Callaghan MJ, Edelstein D, Brownlee M, Gurtner GC. Decreasing intracellular superoxide corrects defective ischemia-induced new vessel formation in diabetic mice. J Biol Chem 2008; 283: 10930-10938.

23. Masoud GN, Li W. HIF-1a pathway: role, regulation and intervention for cancer therapy. Acta Pharm Sin B 2015; 5: 378-389.

24. Kong D, Park EJ, Stephen AG, Calvani M, Cardellina JH, Monks A, Fisher RJ, Shoemaker RH, Melillo G. Echinomycin, a small-molecule inhibitor of hypoxiainducible factor-1 DNA-binding activity. Cancer Res 2005; 65: 9047-9055.

25. Vanhoutte PM, Shimokawa H, Tang EH, Feletou M. Endothelial dysfunction and vascular disease. Acta Physiol (Oxf) 2009; 196: 193-222.

\section{*Correspondence to}

Yiping Liu

Laboratory of Sport Physiology and Biomedicine

School of Physical Education and Sport Sciences

Fujian Normal University

Fuzhou

PR China

Zhengchao Wang

Laboratory of Sport Physiology and Biomedicine

School of Physical Education and Sport Sciences

Fujian Normal University

Fuzhou

PR China 